OPEN ACCESS

Edited by:

Antonella Caputo,

University of Ferrara, Italy

Reviewed by:

Wolfgang Eisenreich,

Technical University of Munich,

Germany

Claudia Claus,

Leipzig University, Germany

*Correspondence: María Maximina Bertha MorenoAltamirano

bertha.moreno.altamirano@gmail.com

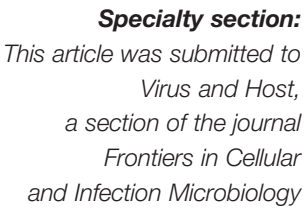

Received: 14 June 2021

Accepted: 16 August 2021

Published: 14 September 2021

Citation:

Sánchez-García FJ,

Pérez-Hernández CA,

Rodríguez-Murillo $M$ and

Moreno-Altamirano MMB (2021)

The Role of Tricarboxylic Acid Cycle

Metabolites in Viral Infections.

Front. Cell. Infect. Microbiol. 11:725043.

doi: 10.3389/fcimb.2021.725043

\title{
The Role of Tricarboxylic Acid Cycle Metabolites in Viral Infections
}

\section{Francisco Javier Sánchez-García, Celia Angélica Pérez-Hernández, Miguel Rodríguez-Murillo and María Maximina Bertha Moreno-Altamirano*}

Laboratorio de Inmunorregulación, Departamento de Inmunología, Escuela Nacional de Ciencias Biológicas, Instituto Politécnico Nacional, Mexico City, Mexico

Host cell metabolism is essential for the viral replication cycle and, therefore, for productive infection. Energy (ATP) is required for the receptor-mediated attachment of viral particles to susceptible cells and for their entry into the cytoplasm. Host cells must synthesize an array of biomolecules and engage in intracellular trafficking processes to enable viruses to complete their replication cycle. The tricarboxylic acid (TCA) cycle has a key role in ATP production as well as in the synthesis of the biomolecules needed for viral replication. The final assembly and budding process of enveloped viruses, for instance, require lipids, and the TCA cycle provides the precursor (citrate) for fatty acid synthesis (FAS). Viral infections may induce host inflammation and TCA cycle metabolic intermediates participate in this process, notably citrate and succinate. On the other hand, viral infections may promote the synthesis of itaconate from TCA cis-aconitate. Itaconate harbors anti-inflammatory, anti-oxidant, and anti-microbial properties. Fumarate is another TCA cycle intermediate with immunoregulatory properties, and its derivatives such as dimethyl fumarate (DMF) are therapeutic candidates for the contention of virus-induced hyper-inflammation and oxidative stress. The TCA cycle is at the core of viral infection and replication as well as viral pathogenesis and anti-viral immunity. This review highlights the role of the TCA cycle in viral infections and explores recent advances in the fast-moving field of virometabolism.

Keywords: viruses, host cell metabolism, metabolic reprogramming, mitochondria, tricarboxylic acid cycle

\section{OVERVIEW OF THE TCA CYCLE}

Comprehensive reviews on metabolic pathways, including the TCA cycle and their role beyond metabolism, have recently been published (Claus and Liebert, 2014; O'Neill et al., 2016; Williams and O'Neill, 2018; Martínez-Reyes and Chandel, 2020; Choi et al., 2021). In brief, the TCA cycle (also known as Krebs cycle or citric acid cycle) takes place within mitochondria and initiates with a reaction catalyzed by the enzyme citrate synthase, leading to the synthesis of citrate from the condensation of oxaloacetate with acetyl-coenzyme-A. The latter is produced outside the mitochondria from the catabolism of carbohydrates, proteins, and fatty acids, as well as from the breakdown of the citrate exported from mitochondria to the cytosol. From citrate, the TCA cycle proceeds through eight enzymatic reactions whereby citrate is converted to cis-aconitate, cisaconitate to isocitrate, isocitrate to $\alpha$-ketoglutarate, $\alpha$-ketoglutarate to succinyl-CoA, which is then converted to succinate, succinate to fumarate, fumarate to malate and finally, malate to oxaloacetate which upon condensation with acetyl-coenzyme-A initiates a new TCA cycle. 
The TCA cycle is linked to the electron transport chain (ETC) at the succinate dehydrogenase $\mathrm{SDH}$, mitochondrial respiratory complex II). The TCA cycle produces NADH (nicotinamide adenine dinucleotide, in its reduced form) and FADH2 (flavin adenine dinucleotide, in its reduced form), which are oxidized by the mitochondrial ETC for the synthesis of ATP.

Metabolic diversions from the TCA cycle may take place and in this way citrate can exit from the TCA cycle and be exported to the cytoplasm in exchange for malate by the family 25 member 1 (Slc25a1) solute carrier.

Cytosolic citrate is converted into oxaloacetate and acetyl-CoA, which is converted into malonyl-coenzyme-A to be incorporated into cholesterol or fatty acids (Williams and O'Neill, 2018). Likewise, cis-aconitate can be diverted from the synthesis of isocitrate to produce itaconate instead, by means of the cis-aconitate decarboxylase enzyme, the product of the immune-responsive gene 1 ( $\operatorname{Irg} 1$ ), latter renamed as Acod1 (Williams and $\mathrm{O}^{\prime} \mathrm{Neill}$, 2018). Itaconate is an inhibitor of $\mathrm{SDH}$, inhibiting the conversion of succinate into fumarate (Figure 1).

\section{TCA CYCLE AND VIRUS REPLICATION CYCLE}

The cross-talk between host cell metabolism and viruses may be approached from different but not excluding perspectives: 1) metabolism and virus replication cycle 2) metabolism and anti- viral immunity 3) virus control of immune cells metabolism for the subversion of anti-viral immune responses 4) metabolism and inhibition of viral replication, and 5) metabolite-dependent viral pathogenicity (Lewis and Scott, 1962; Landini, 1984; Maynard et al., 2010; Yu et al., 2011; Sánchez and Lagunoff, 2015; Netea et al., 2016; Eisenreich et al., 2019; Mayer et al., 2019; Moreno-Altamirano et al., 2019; Olagnier et al., 2020).

Pioneering work analyzing the role of host cell metabolism on viral replication goes back to the early 1950s when Ackermann et al. first observed that ex vivo influenza A virus infection of mice tissues was impaired by the addition of chemical inhibitors of TCA cycle enzymes (Ackermann et al., 1951).

Viruses require that their host cells synthesize lipids, proteins and nucleic acids to ensure a viral progeny, and that they produce energy to drive viral assembly and release (Hui and Nayak, 2001; Claus and Liebert, 2014). Fatty acid synthesis requires glycolysis, pentose phosphate pathway, and TCA cycle metabolic intermediates. TCA cycle-derived citrate may be exported out of the mitochondria towards the cytosol where it is converted to acetyl-CoA by the ATP citrate lyase enzyme and then to malonyl-CoA by the acetyl-CoA carboxylase enzyme, from malonyl-CoA, the elongation of straight-chain fatty acids proceeds sequentially by means of the fatty acid synthase, in an $\mathrm{NADPH}$-dependent reaction, up to the formation of palmitate (C16); further elongation and the insertion of double bonds require other enzyme systems. The synthesis of branched-chain fatty acid requires branched-chain amino acids as substrates, and triacylglycerols and phospholipids are synthesized from the

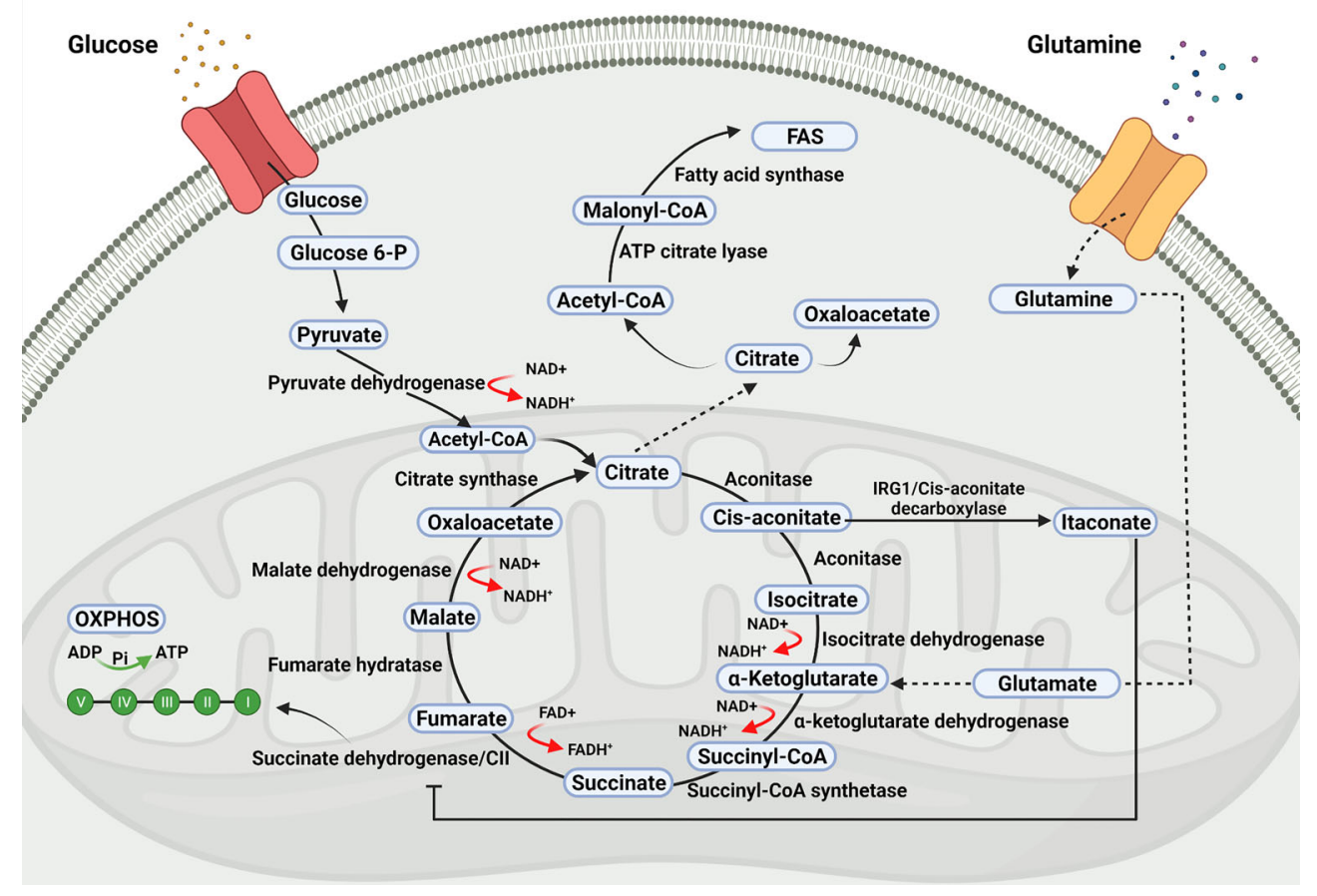

FIGURE 1 | Tricarboxylic acid (TCA) cycle. The TCA cycle takes place within the mitochondria and it is the source of precursors for the synthesis of biomolecules required for viral replication as well as of metabolites with signalling properties which may influence the outcome of viral infections. 
condensation of fatty acids with glycolysis-derived glycerol (O’Neill et al., 2016).

Glutamine is converted to glutamate, which is incorporated into the TCA cycle as $\alpha$-ketoglutarate, providing the precursors for aspartic acid, asparagine, glutamic acid, and proline, as well as citrate. The cell uptake of glutamine allows the flux of glucose to the pentose-phosphate pathway and, amino acid, nucleotide, and fatty acids biosynthesis (Boroughs and DeBerardinis, 2015).

Viral replication is highly dependent on glucose, glutamine, and the TCA cycle (Mayer et al., 2019). Therefore, how viruses might take control of those metabolic substrates and subvert the TCA cycle to support their replication cycle seems to be a fundamental question. In this regard, the herpes simplex virus1-infected cells may shift glucose metabolism to nucleotide synthesis instead of glycolysis or the TCA cycle, whereas glutamine feeds the TCA cycle, through the synthesis of $\alpha$ ketoglutarate (Vastag et al., 2011; Mayer et al., 2019). Other viruses induce glycolysis or are benefited from a glycolytic cell host, these include dengue virus (DENV) (Fontaine et al., 2015), hepatitis C virus (HCV) (Ramiere et al., 2014), human immunodeficiency virus (HIV) (Palmer et al., 2014), human adenovirus-2 (HAdV-2) (Guissoni et al., 2018), Zika virus (ZIKV) (Singh et al., 2020), and SARS-CoV-2 (Bojkova et al., 2020).

Human adenovirus HAdV-2 infection increases glucose consumption and glycolysis, as well as glutaminolysis, which replenish TCA cycle, whereas the TCA cycle itself undergoes a 1.5 fold increase in the production of metabolic intermediates (Carinhas et al., 2017). Proteomic analysis of cells infected with HAdV-2 showed the up-regulation of glycolysis and TCA cycle enzymes, along with those of other metabolic pathways (Guissoni et al., 2018). Zika virus infection up-regulates several genes involved in glycolysis, including the glucose transporter 1 (GLUT1), and the monocarboxylate transporter MCT4, which expulse lactate outside cells (Singh et al., 2020). Moreover, inhibition of glycolysis impairs replication of herpes simplex virus (Courtney et al., 1973; Mayer et al., 2019), and SARS-CoV2 (Bojkova et al., 2020).

In addition to their role as a source of energy and biomolecule precursors for virus assembly, TCA metabolites may serve signaling roles (Williams and O'Neill, 2018; Hooftman et al., 2020), all of which open new avenues for the understanding of host cell-virus interaction, and the view of cell metabolism as a possible target for anti-viral intervention.

\section{TCA INTERMEDIATE METABOLITES WITH KNOWN SIGNALING PROPERTIES}

The TCA cycle metabolic intermediates, citrate, succinate, and fumarate, are known to harbor signaling properties (McGettrick and O'Neill, 2013; Mills and O'Neill, 2014; Williams and O'Neill, 2018). In addition, itaconate, a derivative from TCA cycle cisaconitate is also a signaling molecule (O'Neill and Artyomov, 2019; Hooftman et al., 2020).

\section{CITRATE AND ITS ROLE IN VIRAL INFECTION}

The mitochondrial citrate carrier exports citrate to the cytosol to be converted into acetyl-CoA and oxaloacetate. A key role of acetyl-CoA in the inflammatory process is the acetylation of histones, leading to the increased synthesis of IL- $1 \beta$, TNF- $\alpha$, and IFN- $\gamma$ (Ashbrook et al., 2015; Mills et al., 2017; Williams and O’Neill, 2018).

Citrate links carbohydrate and fatty acid metabolism. Fatty acid synthesis is necessary for virus replication since it facilitates viral entry, replication, and egress from host cells (Chukkapalli et al., 2012). Most viruses replicate in specific membranous compartments, which differ from virus to virus. Flaviviruses, for instance, actively regulate lipid biosynthesis to establish their replication sites (Rothwell et al., 2009; Heaton et al., 2010; Chukkapalli et al., 2012). The dependence on fatty acid synthesis has been studied in several other viruses including dengue, West Nile, and hepatitis C (Heaton and Randall, 2011; Alvisi et al., 2011; Zhang et al., 2016; Starting and van Kuppeveld, 2017).

Citrate-derived acetyl-CoA has a role in eukaryotic histone acetylation that can be regulated by viral infections (Lu et al., 2003; Hancock et al., 2010). In addition, acetyl-CoA participates in the acetylation of non-histone proteins (Choudhary et al., 2009; Christensen et al., 2019). Protein acetylation is a prevalent lysine post-transcriptional modification that regulates epigenetics (histone acetylation) and protein function (nonhistone acetylation). As a way of an example, NIH3T3 cells infected with Moloney murine leukemia virus and treated with histone deacetylase (HDAC) inhibitors increase the expression of unintegrated retroviral DNA, without increasing the amount of integrated DNA. First supporting the notion that unintegrated DNAs are functionally associated with nucleosomes and secondly that histone acetylation increases the stability of unintegrated DNA, thus increasing the viral template availability for transcription (Wang et al., 2016).

On the other hand, some viruses might regulate the acetyation of several host proteins contributing to host antiviral response as well as to viral replication (Murray et al., 2018). In this way, the interferon inducible protein IFI16 is acetylated within its nuclear localization motif, determining its cellular location and therefore its ability to initiate anti viral DNA innate immune response (Li et al., 2012).

Several viral proteins are the targets of acetylation, a process that regulates virus cellular location, viral transcriptional activities, and virulence, such proteins include the HIV Tat protein, the Kaposi's sarcoma-associated herpesvirus latencyassociated nuclear antigen, the human papillomavirus E2 protein, the H1N1 influenza virus NS1 protein, and the hepatitis D virus and influenza virus nucleocapsids (Ott et al., 1999; Mu et al., 2004; Lu et al., 2006; Quinlan et al., 2013; Hatakeyama et al., 2018; Ma et al., 2020) (Figure 2). Acetylome analyzes during in vitro infection with human cytomegalovirus have showed that amongst the proteins that display increased acetylation are the pyruvate dehydrogenase 


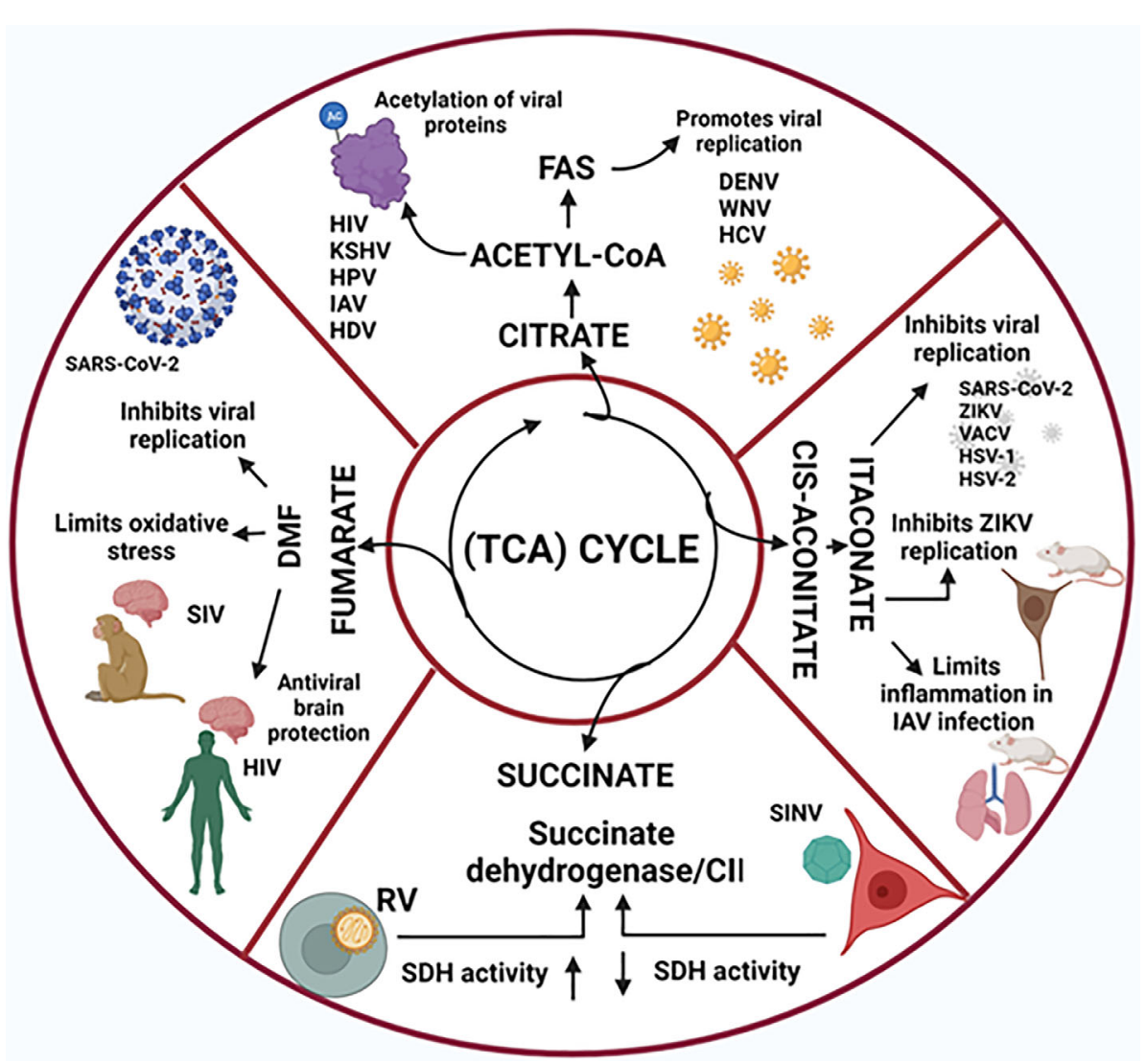

FIGURE 2 | Tricarboxylic acid (TCA) cycle-derived metabolites and their role in viral infections. The TCA cyce intermediate metabolites citrate, succinate and fumarate play key roles in vral replication and in the virus-induced inflammation process. In addition, itaconate, which is synthesized from the TCA cis-aconitate, is also endowed with anti-inflammatory and anti-viral properties.

(E1), dihydrolipoamide S-acetyltransferase (E2), and the dihydrolipoamide dehydrogenase (E3) components of the pyruvate dehydrogenase complex, which catalyzes the oxidation of pyruvate to acetyl-CoA and $\mathrm{CO}_{2}$ and interestingly, the dynamic translocation of the mitochondrial pyruvate dehidrogenase complex to the nucleus ensures the nuclear production of acetyl-CoA necessary for histone acetylation and epigenetic regulation (Murray et al., 2018).

Specific viral proteins might regulate the TCA cycle. The vaccinia virus for instance, encodes a viral homolog of the epidermal growth factor called viral growth factor which binds to the epithelial growth factor receptor, activates MAPK signaling and phosphorylates the signal transducer and activator of transcription 3 (STAT3) at the non-canonical site serine727. In this way vaccinia virus induces a 3.3 -fold increase in the production of citrate and other TCA cycle metabolic intermediates at $8 \mathrm{~h}$ post infection (Pant et al., 2021), thus contributing to fatty acid synthesis and protein acetylation. Since protein acetylation regulates enzyme activity, chromatin structure, protein localization, and protein-protein interaction (Murray et al., 2018), the function of specific acetylated proteins and its impact on viral replication cycle remains to be analyzed.

\section{ITACONATE AND ITS ROLE IN VIRAL INFECTION}

By using integrated high throughput transcriptional-metabolic profiling during murine macrophage M1 polarization, Jha et al. (Jha et al., 2015) analyzed a TCA cycle break at isocitrate dehydrogenase, the metabolic step that converts isocitrate to $\alpha$ ketoglutarate, and demonstrated that in the TCA broken cycle, citric acid is redirected to itaconic acid synthesis, by the upregulation of the immunoresponsive gene 1 (Irg-1, currently known as Acod-1), which encodes the cis-aconitate decarboxylase enzyme that catalyzes the conversion of cisaconitate to itaconate (Michelucci et al., 2013; Németh et al., 2016).

Itaconate inhibits $\mathrm{SDH}$, thus regulating succinate levels, mitochondrial respiration and the production of proinflammatory cytokines (Cordes et al., 2016; Lampropoulou et al., 2016; Cordes and Metallo, 2021). In addition, itaconate alkylates cysteine residues in the KEAP1 (Kelch-like ECHassociated protein 1) protein, which normally associates with Nrf2 (Nuclear factor erythroid 2 (NF-E2)-related factor 2), promoting its degradation. KEAP1 alkylation prevents Nrf2 
degradation, allowing its accumulation, nuclear translocation, and the transcriptional activation of anti-oxidant and antiinflammatory programs (Hayes and Dinkova-Kostova, 2014; Mills et al., 2018). An extracellular source of itaconate or its derivatives may regulate succinate levels, type I interferons, and reduce the expression of pro-IL-1 $\beta$, IL-6, IL-12, and iNOS in macrophages (Lampropoulou et al., 2016; Swain et al., 2020). Itaconate may modify target proteins at cysteine residues, contributing to its role as an immunomodulator (Hooftman and O'Neil, 2019). On the other hand, itaconate induces electrophilic stress and inhibition of the $I \kappa B \zeta$-mediated

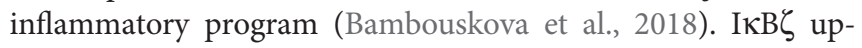
regulates the expression of secondary response genes that enhance the TLR/IL-1R signalling pathways leading to cytokine production (Yamamoto et al., 2004; Ohto-Ozaki et al., 2020).

The itaconate derivative 4-octyl-itaconate (4-OI) limits the SARS-CoV-2 infection- associated host inflammatory response while inhibiting SARS- CoV-2 replication, independently of type I interferon (IFN) signaling (Olagnier et al., 2020), 4-OI also inhibits the replication of herpes simplex virus-1 and-2 (HSV-1 and $\mathrm{HSV}-2$ ), vaccinia virus (VACV), and Zika virus (Olagnier et al., 2020).

Infection of mice with influenza A virus induces the expression of Acod- 1 and the synthesis of itaconate which limits pulmonary inflammation and disease severity, whereas treatment with exogenous itaconate or its derivative dimethyl-itaconate reduces IFN responses and down-modulate pro-inflammatory cytokines, also limiting inflammation (Sohail et al., 2021). On the other hand, the itaconate derivative 4-octyl-itaconate suppresses influenza A virus replication in PBMC (Sohail et al., 2021). Thus itaconate and its derivatives limit inflammation and related pathologies, and suppresses viral replication.

Previously, using a murine model of ZIKV infection, Daniels et al. had shown that ZIKV activates a signaling pathway involving a the receptor interacting protein kinase-3 (RIPK3), leading to the up-regulation of Acod-1 and the production of itaconate, which suppresses the replication of ZIKV in neurons (Daniels et al., 2019).

It was recently shown that the expression of the Nrf2 gene which regulates the transcription of the glutathione and thioredoxin anti-oxidant systems, detoxification, NADPH regeneration, and heme metabolism (Tonelli et al., 2018) is suppressed in biopsies obtained from COVID-19 patients (Olagnier et al., 2020). In addition, itaconate induces electrophilic stress and inhibition of the $I \kappa B \zeta$-mediated

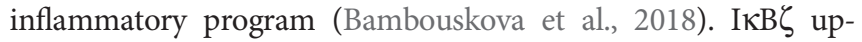
regulates the expression of secondary response genes that enhance the TLR/IL-1R signalling pathways leading to cytokine production (Yamamoto et al., 2004; Ohto-Ozaki et al., 2020).

In addition to its anti-oxidant and anti-inflammatory roles, Nrf2 can also regulate the sensing of viral DNA in the cytoplasm and thus the release of antiviral type I IFNs, by decreasing STING mRNA stability, linking itaconate synthesis (or cell treatment with the itaconate derivative 4-O-I) with antiviral cytosolic DNA sensing (Olagnier et al., 2018) (Figure 2).

\section{SUCCINATE AND ITS ROLE IN VIRAL INFECTION}

Lipopolysaccharide (LPS) activation of macrophages shifts cell metabolism to glycolytic ATP production, releasing mitochondria from ATP production enabling the increase of mitochondrial membrane potential and succinate synthesis, which oxidation by SDH, leads macrophages to an inflamatory state. The stimulation of dendritic cells and macrophages with LPS drives the succinylation of glyceraldehyde 3-phosphate dehydrogenase (GAPDH), lactate dehydrogenase (LDH), malate dehydrogenase $(\mathrm{MDH})$ and glutamate carrier 1 (Tannahill et al., 2013; Mills et al., 2016).

Succinate is a pro-inflammatory metabolite as well as a competitive inhibitor of various $\alpha$-ketoglutarate-dependent dioxygenases, such as histone demethylases and prolyl hydroxylases. The succinate receptor SUCNR1/GPR91, might signal in synergy with Toll-like receptor 3 and Toll-like receptor 7 to increase the synthesis of pro-inflammatory cytokines, whereas the inhibition of histone demethylases and prolyl hydroxylases contributes to the epigenetic regulation (Rubic et al., 2008; Xiao et al., 2012).

Whether virus-induced glycolysis is associated with other traits of cell activation-induced glycolysis, such as succinate metabolism, requires further analysis. However, there are some instances in which SDH activity is altered during viral infection (Escoll et al., 2019). Infection of three different human cell lines with rubella virus strongly increases SDH activity, along with a slight increase of respiratory complex III activity and decreased activity of complex IV (Claus et al., 2013; Escoll et al., 2019), whereas infection of a mouse neuroblastoma cell line with the Sindbis virus decreases SDH activity, along with respiratory complex I (Silva da Costa et al., 2012; Escoll et al., 2019).

While the role of succinate as a signal in the communication between host cells and pathogenic bacteria is being unveiled (Rosenberg et al., 2021), the role of succinate on viral infections is less studied (Figure 2).

\section{FUMARATE AND ITS ROLE IN VIRAL INFECTION}

Fumarate is the product of the oxidation of succinate, by the succinate dehydrogenase enzyme. Fumarate and its derivatives monomethyl fumarate (MMF) and dimethyl fumarate (DMF) are potent immunomodulators and anti-oxidants that activate Nrf2 (Cecchini, 2003; Wilms et al., 2010; Cross et al., 2011; Linker et al., 2011). DMF inhibits maturation of dendritic cells (DCs) (Peng et al., 2012) and drives the production of IL-10, IL12 and IL-23 by DCs thus down-regulating pathogenic T lymphocytes (Schlöder et al., 2017), DMF also inhibits Th1 to Th2 lymphocyte transition, pro-inflammatory cytokine signaling, the nuclear translocation of the transcription factor $\mathrm{NF}-\mathrm{kB}$, and the expression of cell adhesion molecules in lymphocytes and endothelial cells (Rubant et al., 2008; Gillard et al., 2015; McGuire et al., 2016; Wu et al., 2017). 
DMF has been approved for the treatment of multiple sclerosis and might also protect against systemic and central nervous system complications in HIV infection (Gill and Kolson, 2013). In a study on rhesus macaques infected with simian immunodeficiency virus, treatment with DMF limited the oxidative stress throughout the brain (Garcia-Mesa et al., 2021). Olagnier et al. demonstrated that DMF inhibits SARS$\mathrm{CoV}-2$ replication and the expression of associated inflammatory genes, thus proposing that DMF could be repurposed as a small molecule inhibitor of SARS-CoV-2 replication and inflammation-associated pathology in COVID-19 patients (Olagnier et al., 2020; Timpani and Rybalka, 2021) (Figure 2). It is likely that inflammatory processes associated with other viral infections could also be treated with DMF.

As a note of caution, the use of DMF induces lymphopenia in about $3 \%$ of the patients so treated for relapsing-remitting multiple sclerosis, and at least one case of herpes simplex encephalitis associated with DMF treatment has been reported (Perini et al., 2018).

\section{CONCLUDING REMARKS}

In addition to their role in biosynthesis and energy production which are critial for viral infections, the TCA cycle metabolites citrate, succinate and fumarate, as well as itaconate, which is synthesized from the TCA cycle cis-aconitate, play key roles in the pro-inflammatory/anti-inflammatory homeostasis, contributing to both anti-viral immune response and virusinduced inflammation and associated pathologies. The study of these metabolites in the context of viral infections offers the opportunity to understand better the host cell-virus interaction and to develop new pathophysiological assessment tools and anti-viral therapeutics.

The use of serum concentrations of TCA metabolites as biomarkers of infection and pathology is a promising

\section{REFERENCES}

Ackermann, W. W., Kleinschmidt, E., and Kurtz, H. (1951). The Relation of the Krebs Cycle to Viral Synthesis II. The Effect of Sodium Fluoroacetate on the Propagation of Influenza Virus in Mice. J. Exp. Med. 93, 635-642. doi: 10.1084/ jem.93.6.635

Alvisi, G., Madan, V., and Bartenschlager, R. (2011). Hepatitis C Virus and Host Cell Lipids: An Intimate Connection. RNA Biol. 8, 258-269. doi: 10.4161/rna.8.2.15011

Ashbrook, M. J., McDonough, K. L., Pituch, J. J., Christopherson, P. L., Cornell, T. T., Selewski, D. T., et al. (2015). Citrate Modulates Lipopolysaccharide-Induced Monocyte Inflammatory Responses. Clin. Exp. Immunol. 180, 520-530. doi: $10.1111 /$ cei.12591

Bambouskova, M., Gorvel, L., Lampropoulou, V., Sergushichev, A., Loginicheva, E., Johnson, K., et al. (2018). Electrophilic Properties of Itaconate and Derivatives Regulate the Iאbל-ATF3 Inflammatory Axis. Nature. 556, 501504. doi: 10.1038/s41586-018-0052-Z

Beloborodova, N., Pautova, A., Sergeev, A., and Fedotcheva, N. (2019). Serum Levels of Mitochondrial and Microbial Metabolites Reflect Mitochondrial Dysfunction in Different Stages of Sepsis. Metabolites 9, 196. doi: 10.3390/ metabo9100196

Bojkova, D., Klann, K., Koch, B., Widera, M., Krause, D., Ciesek, S., et al. (20202020). Proteomics of SARS-CoV-2-Infected Host Cells Reveals Therapy Targets. Nature 583, 469e472. doi: 10.1038/s41586-020-2332-7 approach. The serum concentrations of succinate, $\alpha$ ketoglutarate, fumarate and itaconate, for instance, have been used for the clinical follow up of sepsis (Beloborodova et al., 2019) and more recently the serum metabolome of COVID-19 patients has informed that succinate levels are higher and citrate levels are lower, as compared with healthy controls, suggesting that these two metabolites along with D-fructose and 2palmitoyl-glycerol are of value in the pathogenesis and diagnosis of COVID-19 (Shi et al., 2021).

The specific role of TCA cycle metabolites on the infection process with particular virus species is being unveiled, and comparative analyses will advance our understanding of virus biology as well as virus-host cell interaction. Finally, the use of TCA metabolites and their derivatives as anti-inflammatory, anti-oxidant, and anti-viral molecules, are paving the way to metabolism-targeted anti-viral therapeutic intervention. We foresee a rapid development in this area.

\section{AUTHOR CONTRIBUTIONS}

FS-G: Conceived the review, wrote the manuscript and approved final version. CP-H: Contributed with writing, review and approved final version MR-M: Contributed with figures design, review and approved final version MM-A: Conceived the review, wrote the manuscript and approved final version. All authors contributed to the article and approved the submitted version.

\section{ACKNOWLEDGMENTS}

We thank Secretaria de Investigación y Posgrado (SIP-20210917) and COFAA-IPN. Figures were created with BioRender.com.

Boroughs, L. K., and DeBerardinis, R. J. (2015). Metabolic Pathways Promoting Cancer Cell Survival and Growth. Nat. Cell Biol. 17, 351-359. doi: 10.1038/ ncb3124

Carinhas, N., Koshkin, A., Pais, D. A. M., Alves, P. M., and Teixeira, A. P. (2017). 13C Metabolic Flux Analysis of Human Adenovirus Infection: Implications for Viral Vector Production. Biotechnol. Bioeng. 114, 195-207. doi: 10.1002/bit.26063

Cecchini, G. (2003). Function and Structure of Complex II of the Respiratory Chain. Annu. Rev. Biochem. 72, 77-109. doi: 10.1146/annurev.biochem.72.121801.161700

Choi, I., Son, H., and Baek, J.-H. (2021). Tricarboxylic Acid (TCA) Cycle Intermediates: Regulators of Immune Responses. Life 11, 69. doi: 10.3390/life11010069

Choudhary, C., Kumar, C., Gnad, F., Nielsen, M. L., Rehman, M., Walther, T. C., et al. (2009). Lysine Acetylation Targets Protein Complexes and Co-Regulates Major Cellular Functions. Science 325, 834-840. doi: 10.1126/science.1175371

Christensen, D. G., Xie, X., Basisty, N., Byrnes, J., McSweeney, S., Schilling, B., et al. (2019). Post-Translational Protein Acetylation: An Elegant Mechanism for Bacteria to Dynamically Regulate Metabolic Functions. Front. Microbiol. 10, 1604. doi: 10.3389/fmicb.2019.01604

Chukkapalli, V., Heaton, N. S., and Randall, G. (2012). Lipids at the Interface of Virus-Host Interactions. Curr. Opin. Microbiol. 15, 512-518. doi: 10.1016/ j.mib.2012.05.013

Claus, C., and Liebert, U. G. A. (2014). Renewed Focus on the Interplay Between Viruses and Mitochondrial Metabolism. Arch. Virol. 159, 1267-1277. doi: 10.1007/s00705-013-1841-1 
Claus, C., Schonefeld, K., Hubner, D., Chey, S., Reibetanz, U., and Liebert, U. G. (2013). Activity Increase in Respiratory Chain Complexes by Rubella Virus With Marginal Induction of Oxidative Stress. J. Virol. 87, 8481-8492. doi: 10.1128/JVI.00533-13

Cordes, T., and Metallo, C. M. (2021). Itaconate Alters Succinate and Coenzyme A Metabolism via Inhibition of Mitochondrial Complex II and MethylmalonylCoA Mutase. Metabolites 11, 117. doi: 10.3390/metabo11020117

Cordes, T., Wallace, M., Michelucci, A., Divakaruni, A. S., Sapcariu, S. C., Sousa, C., et al. (2016). Immunoresponsive Gene 1 and Itaconate Inhibit Succinate Dehydrogenase to Modulate Intracellular Succinate Levels. J. Biol. Chem. 291, 14274-14284. doi: 10.1074/jbc.M115.685792

Courtney, R. J., Steiner, S. M., and Benyesh-Melnick, M. (1973). Effects of 2Deoxy-D-Glucose on Herpes Simplex Virus Replication. Virol. 52, 447-455. doi: 10.1016/0042-6822(73)90340-1

Cross, S., Cook, D. R., Chi, A. W., Vance, P. J., Kolson, L. L., Wong, B. J., et al. (2011). Dimethyl Fumarate, an Immune Modulator and Inducer of the Antioxidant Response, Suppresses HIV Replication and MacrophageMediated Neurotoxicity: A Novel Candidate for HIV Neuroprotection. J. Immunol. 187, 5015-5025. doi: 10.4049/jimmunol.1101868

Daniels, B. P., Kofman, S. B., Smith, J. R., Norris, G. T., Snyder, A. G., Kolb, J. P., et al. (2019). The Nucleotide Sensor ZBP1 and Kinase RIPK3 Induce the Enzyme IRG1 to Promote an Antiviral Metabolic State in Neurons. Immunity 50, 64-76. doi: 10.1016/j.immuni.2018.11.017

Eisenreich, W., Rudel, T., Heesemann, J., and Goebel, W. (2019). How Viral and Intracellular Bacterial Pathogens Reprogram the Metabolism of Host Cells to Allow Their Intracellular Replication. Front. Cell. Infect. Microbiol. 9, 42. doi: 10.3389/fcimb.2019.00042

Escoll, P., Platon, L., and Buchrieser, C. (2019). Roles of Mitochondrial Respiratory Complexes During Infection. Immunometabolism 1, e190011. doi: 10.20900/immunometab20190011

Fontaine, K. A., Sanchez, E. L., Camarda, R., and Lagunoff, M. (2015). Dengue Virus Induces and Requires Glycolysis for Optimal Replication. J. Virol. 89, 2358-2366. doi: 10.1128/JVI.02309-14

Garcia-Mesa, Y., Xu, H. N., Vance, P., Gruenewald, A. L., Garza, R., Midkiff, C., et al. (2021). Dimethyl Fumarate, an Approved Multiple Sclerosis Treatment, Reduces Brain Oxidative Stress in SIV-Infected Rhesus Macaques: Potential Therapeutic Repurposing for HIV Neuroprotection. Antioxidants. Basel. 10, 416. doi: 10.3390/antiox10030416

Gillard, G. O., Collette, B., Anderson, J., Chao, J., Scannevin, R. H., Huss, D. J., et al. (2015). DMF, But Not Other Fumarates, Inhibits NF-kappaB Activity In Vitro in an Nrf2-Independent Manner. J. Neuroimmunol. 283, 74-85. doi: 10.1016/j.jneuroim.2015.04.006

Gill, A. J., and Kolson, D. L. (2013). Dimethyl Fumarate Modulation of Immune and Antioxidant Responses: Application to HIV Therapy. Crit. Rev. Immunol. 33, 307-359. doi: 10.1615/critrevimmunol.2013007247

Guissoni, A. C. P., Soares, C. M. A., Badr, K. R., Ficcadori, F. S., Parente, A. F. A., Parente, J. A., et al. (2018). Proteomic Analysis of A-549 Cells Infected With Human Adenovirus 40 by LC-MS. Virus Genes 54, 351-360. doi: 10.1007/ s11262-018-1554-3

Hancock, M. H., Cliffe, A. R., Knipe, D. M., and Smiley, J. R. (2010). Herpes Simplex Virus VP16, But Not ICP0, Is Required to Reduce Histone Occupancy and Enhance Histone Acetylation on Viral Genomes in U2OS Osteosarcoma Cells. J. Virol. 84, 1366-1375. doi: 10.1128/JVI.01727-09

Hatakeyama, D., Shoji, M., Yamayoshi, S., Yoh, R., Ohmi, N., Takenaka, S., et al. (2018). Influenza A Virus Nucleoprotein Is Acetylated by Histone Acetyltransferases PCAF and GCN5. J. Biol. Chem. 293, 7126-77138. doi: $10.1074 /$ jbc.RA117.001683

Hayes, J. D., and Dinkova-Kostova, A. T. (2014). The Nrf2 Regulatory Network Provides an Interface Between Redox and Intermediary Metabolism. Trends Biochem. Sci. 39, 199-218. doi: 10.1016/j.tibs.2014.02.002

Heaton, N. S., Perera, R., Berger, K. L., Khadka, S., Lacount, D. J., Kuhn, R. J., et al. (2010). Dengue Virus Nonstructural Protein 3 Redistributes Fatty Acid Synthase to Sites of Viral Replication and Increases Cellular Fatty Acid Synthesis. Proc. Natl. Acad. Sci. U. S. A. 107, 17345-17350. doi: 10.1073/ pnas. 1010811107

Heaton, N. S., and Randall, G. (2011). Multifaceted Roles for Lipids in Viral Infection. Trends. Microbiol. 19, 368-375. doi: 10.1016/j.tim.2011.03.007
Hooftman, A., and O'Neill, L. A. J. (2019). The Immunomodulatory Potential of the Metabolite Itaconate. Trends Immunol. 40, 687-698. doi: 10.1016/ j.it.2019.05.007

Hooftman, A., Angiari, S., Hester, S., Corcoran, S. E., Runtsch, M. C., Ling, C., et al. (2020). The Immunomodulatory Metabolite Itaconate Modifies NLRP3 and Inhibits Inflammasome Activation. Cell. Metab. 32, 468-478. doi: 10.1016/ j.cmet.2020.07.0168-478

Hui, E. K., and Nayak, D. P. (2001). Role of ATP in Influenza Virus Budding. Virology 290, 329-341. doi: 10.1006/viro.2001.1181

Jha, A. K., Huang, S. C.-C., Sergushichev, A., Lampropoulou, V., Ivanova, Y., Loginicheva, E., et al. (2015). Network Integration of Parallel Metabolic and Transcriptional Data Reveals Metabolic Modules That Regulate Macrophage Polarization. Immunity 42, 419-430. doi: 10.1016/j.immuni.2015.02.005

Lampropoulou, V., Sergushichev, A., Bambouskova, M., Nair, S., Vincent, E. E., Loginicheva, E., et al. (2016). Itaconate Links Inhibition of Succinate Dehydrogenase With Macrophage Metabolic Remodeling and Regulation of Inflammation. Cell. Metab. 24, 158-166. doi: 10.1016/j.cmet.2016.06.004

Landini, M. P. (1984). Early Enhanced Glucose Uptake in Human Cytomegalovirus-Infected Cells. J. Gen. Virol. 65, 1229-1232. doi: 10.1099/ 0022-1317-65-7-1229

Lewis, V. J., and Scott, L. V. (1962). Nutritional Requirements for the Production of Herpes Simplex Virus. I. Influence of Glucose and Glutamine of Herpes Simplex Virus Production by HeLa Cells. J. Bacteriol. 83, 475-482. doi: $10.1128 / \mathrm{jb}$.83.3.475-482.1962

Li, T., Diner, B. A., Chen, J., and Cristea, I. M. (2012). Acetylation Modulates Cellular Distribution and DNA Sensing Ability of Interferon-Inducible Protein IFI16. Proc. Natl. Acad Sci. U. S. A. 109, 10558-10563. doi: 10.1073/ pnas.1203447109

Linker, R. A., Lee, D. H., Ryan, S., van Dam, A. M., Conrad, R., Bista, P., et al. (2011). Fumaric Acid Esters Exert Neuroprotective Effects in Neuroinflammation via Activation of the Nrf2 Antioxidant Pathway. Brain 134, 678-692. doi: 10.1093/brain/awq386

Lu, F., Day, L., Gao, S. J., and Lieberman, P. M. (2006). Acetylation of the LatencyAssociated Nuclear Antigen Regulates Repression of Kaposi's SarcomaAssociated Herpesvirus Lytic Transcription. J. Virol. 80, 5273-5282. doi: 10.1128/JVI.02541-05

Lu, F., Zhou, J., Wiedmer, A., Madden, K., Yuan, Y., and Lieberman, P. M. (2003). Chromatin Remodeling of the Kaposi's Sarcoma-Associated Herpesvirus ORF50 Promoter Correlates With Reactivation From Latency. J. Virol. 77, 11425-11435. doi: 10.1128/jvi.77.21.11425-11435.2003

Martínez-Reyes, I., and Chandel, N. S. (2020). Mitochondrial TCA Cycle Metabolites Control Physiology and Disease. Nat. Commun. 11, 102. doi: 10.1038/s41467-019-13668-3

Ma, J., Wu, R., Xu, G., Yuqiang, C., Zhaofei, W., Heng'an, W., et al. (2020). Acetylation at K108 of the NS1 Protein Is Important for the Replication and Virulence of Influenza Virus. Vet. Res. 51, 20. doi: 10.1186/s13567-020-00747-3

Mayer, K. A., Stöckl, J., Zlabinger, G. J., and Gualdoni, G. A. (2019). Hijacking the Supplies: Metabolism as a Novel Facet of Virus-Host Interaction. Front. Immunol. 10, 1533. doi: 10.3389/fimmu.2019.01533

Maynard, N. D., Gutschow, M. V., Birch, E. W., and Covert, M. W. (2010). The Virus as Metabolic Engineer. Biotechnol. J. 5, 686-694. doi: 10.1002/ biot. 201000080

McGettrick, A. F., and O’Neill, L. A. J. (2013). How Metabolism Generates Signals During Innate Immunity and Inflammation. J. Biol. Chem. 288, 22893-22898. doi: $10.1074 /$ jbc.R113.486464

McGuire, V. A., Ruiz-Zorrilla, D. T., Emmerich, C. H., Strickson, S., Ritorto, M. S., Sutavani, R. V., et al. (2016). Dimethyl Fumarate Blocks Pro-Inflammatory Cytokine Production via Inhibition of TLR Induced M1 and K63 Ubiquitin Chain Formation. Sci. Rep. 6, 31159. doi: 10.1038/srep31159

Michelucci, A., Cordes, T., Ghelfi, J., Pailot, A., Reiling, N., Goldmann, O., et al. (2013). Immune-Responsive Gene 1 Protein Links Metabolism to Immunity by Catalyzing Itaconic Acid Production. Proc. Natl. Acad. Sci. U. S. A. 110, 78250 5. doi: 10.1073/pnas. 1218599110

Mills, E. L., Kelly, B., Logan, A., Costa, A. S. H., Varma, M., Bryant, C. E., et al. (2016). Succinate Dehydrogenase Supports Metabolic Repurposing of Mitochondria to Drive Inflammatory Macrophages. Cell 167, 457-470.e13. doi: 10.1016/j.cell.2016.08.064 
Mills, E., and O'Neill, L. A. J. (2014). Succinate: A Metabolic Signal in Inflammation. Trends. Cell. Biol. 24, 313-320. doi: 10.1016/j.tcb.2013.11.008

Mills, E. L., Kelly, B., and O'Neill, L. A. J. (2017). Mitochondria Are the Powerhouses of Immunity. Nat. Immunol. 118, 488-498. doi: 10.1038/ni.3704

Mills, E. L., Ryan, D. G., Prag, H. A., Dikovskaya, D., Menon, D., Zaslona, Z., et al. (2018). Itaconate Is an Anti-Inflammatory Metabolite That Activates Nrf2 via Alkylation of KEAP1. Nature. 556, 113-117. doi: 10.1038/nature25986

Moreno-Altamirano, M. M. B., Kolstoe, S. E., and Sánchez-García, F. J. (2019). Virus Control of Cell Metabolism for Replication and Evasion of Host Immune Responses. Front. Cell. Infect. Microbiol. 9, 95. doi: 10.3389/fcimb.2019.00095

Murray, L. A., Sheng, X., and Cristea, I. M. (2018). Orchestation of Protein Acetylation as a Toggle for Cellular Defense and Virus Replication. Nat. Comm. 9, 4967. doi: 10.1038/541467-018-07179

Mu, J. J., Tsay, Y. G., Juan, L. J., Fu, T. F., Huang, W. H., Chen, D. S., et al. (2004). The Small Delta Antigen of Hepatitis Delta Virus Is an Acetylated Protein and Acetylation of Lysine 72 May Influence Its Cellular Localization and Viral RNA Synthesis. Virology 319, 60-70. doi: 10.1016/ j.virol.2003.10.024

Németh, B., Doczi, J., Csete, D., Kacso, G., Ravasz, D., Adams, D, et al. (2016). Abolition of Mitochondrial Substrate-Level Phosphorylation by Itaconic Acid Produced by LPS-Induced Irg1 Expression in Cells of Murine Macrophage Lineage. FASEB J. 30, 286-300. doi: 10.1096/fj.15-279398

Netea, M. G., Joosten, L. A., Latz, E., Mills, K. H., Natoli, G., Stunnenberg, H. G., et al. (2016). Trained Immunity: A Program of Innate Immune Memory in Health and Disease. Science 352 (6284), aaf1098. doi: 10.1096/fj.15-279398

Ohto-Ozaki, H., Hayakawa, M., Kamoshita, N., Maruyama, T., Tominaga, S.-i., et al. (2020). Induction of $\mathrm{IkBz}$ Augments Cytokine and Chemokine Production by IL-33 in Mast Cells. J. Immunol. 204, 2033-2042. doi: 10.4049/jimmunol.1900315

Olagnier, D., Brandtoft, A. M., Gunderstofte, C., Villadsen, N. L., Krapp, C., Thielke, A. L., et al. (2018). Nrf2 Negatively Regulates STING Indicating a Link Between Antiviral Sensing and Metabolic Reprogramming. Nat. Commun. 9, 3506. doi: 10.1038/s41467-018-05861-7

Olagnier, D., Farahani, E., Thyrsted, J., Blay-Cadanet, J., Herengt, A., Idorn, M., et al. (2020). SARS-CoV2-Mediated Suppression of NRF2-Signaling Reveals Potent Antiviral and Anti-Inflammatory Activity of 4-OctylItaconate and Dimethyl Fumarate. Nat. Commun. 11, 4938. doi: 10.1038/ s41467-020-18764-3

O'Neill, L. A. J., and Artyomov, M. N. (2019). Itaconate: The Poster Child of Metabolic Reprogramming in Macrophage Function. Nat. Rev. Immunol. 19, 273-281. doi: 10.1038/s41577-019-0128-5

O’Neill, L. A. J., Kishton, R. J., and Rathmell, J. (2016). A Guide to Immunometabolism for Immunologists. Nat. Rev. Immunol. 16, 553-565. doi: $10.1038 /$ nri.2016.70

Ott, M., Schnölzer, M., Garnica, J., Fischle, W., Emiliani, S., and Rackwitz, H. R. (1999). Acetylation of the HIV-1 Tat Protein by P300 Is Important for its Transcriptional Activity. Curr. Biol. 9, 1489-1492. doi: 10.1016/s0960-9822 (00)80120-7

Palmer, C. S., Ostrowski, M., Gouillou, M., Tsai, L., Yu, D., Zhou, J., et al. (2014). Increased Glucose Metabolic Activity Is Associated With CD4+ T-Cell Activation and Depletion During Chronic HIV Infection. AIDS. 28, :297-:309. doi: 10.1097/QAD.0000000000000128

Pant, A., Dsouza, L., Cao, S., Peng, C., and Yang, Z. (2021). Viral Growth Factorand STAT3 Signaling-Dependent Elevation of the TCA Cycle Intermediate Levels During Vaccinia Virus Infection. PloS Pathog. 17 (2), e1009303. doi: 10.1371/journal.ppat.1009303

Peng, H., Guerau-de-Arellano, M., Mehta, V. B., Yang, Y., Huss, D. J., Papenfuss, T. L., et al. (2012). Dimethyl Fumarate Inhibits Dendritic Cell Maturation via Nuclear Factor kB (NF-Kb) and Extracellular Signal-Regulated Kinase 1 and 2 (ERK1/2) and Mitogen Stress-Activated Kinase 1 (MSK1) Signaling. J. Biol. Chem. 10, 28017-28026. doi: 10.1074/jbc.M112.383380

Perini, P., Rinaldi, F., Puthenparampil, M., Marcon, M., Perini, F., and Gallo, P. (2018). Herpes Simplex Virus Encephalitis Temporally Associated With Dimethyl Fumarate-Induced Lymphopenia in a Multiple Sclerosis Patient. Mult. Scler. Relat. Disord. 26, 68-70. doi: 10.1016/j.msard.2018.09.009

Quinlan, E. J., Culleton, S. P., Wu, S. Y., Chiang, C. M., and Androphy, E. J. (2013). Acetylation of Conserved Lysines in Bovine Papillomavirus E2 by P300. J. Virol. 87, 1497-1507. doi: 10.1128/JVI.02771-12
Ramiere, C., Rodriguez, J., Enache, L. S., Lotteau, V., Andre, P., and Diaz, O. (2014). Activity of Hexokinase is Increased by its Interaction With Hepatitis $C$ Virus Protein NS5A. J. Virol. 88, 3246-3254. doi: 10.1128/JVI.02862-13

Rosenberg, G., Yehezkel, D., Hoffman, D., Mattioli, C. C., Fremder, M., BenArosh, H., et al. (2021). Host Succinate is an Activation Signal for Salmonella Virulence During Intracellular Infection. Science. 371, 400-405. doi: 10.1126/ science.aba 8026

Rothwell, C., Lebreton, A., Young, Ng. C., Lim, J. Y., Liu, W., Vasudevan, S., et al. (2009). Cholesterol Biosynthesis Modulation Regulates Dengue Viral Replication. Virology 389, 8-19. doi: 10.1016/j.virol.2009.03.025

Rubic, T., Lametschwandtner, G., Jost, S., Hinteregger, S., Kund, J., CarballidoPerring, N., et al. (2008). Triggering the Succinate Receptor GPR91 on Dendritic Cells Enhances Immunity. Nat. Immunol. 9, 1261-1269. doi: $10.1038 /$ ni. 1657

Rubant, S. A., Ludwig, R. J., Diehl, S., Hardt, K., Kaufmann, R., Pfeilschifter, J. M., et al. (2008). Dimethylfumarate Reduces Leukocyte Rolling In Vivo Through Modulation of Adhesion Molecule Expression. J. Invest. Dermatol. 128, 326331. doi: 10.1038/sj.jid.5700996

Sanchez, E. L., and Lagunoff, M. (2015). Viral Activation of Cellular Metabolism. Virology 479, 609-618. doi: 10.1016/j.virol.2015.02.038

Schlöder, J., Berges, C., Luessi, F., and Jonuleit, H. (2017). Dimethyl Fumarate Therapy Significantly Improves the Responsiveness of T Cells in Multiple Sclerosis Patients for Immunoregulation by Regulatory T Cells. Int. J. Mol. Sci. 18, 271. doi: 10.3390/ijms18020271

Shi, D., Yan, R., Lv, L., Jiang, H., Lu, Y., Sheng, J., et al. (2021). The Serum Metabolome of COVID-19 Patients Is Distinctive and Predictive. Met. Clin. Exp. 118, 154739. doi: 10.1016/j.metabol.2021.154739

Silva da Costa, L., Pereira da Silva, A. P., Da Poian, A. T., and El-Bacha, T. (2012). Mitochondrial Bioenergetic Alterations in Mouse Neuroblastoma Cells Infected With Sindbis Virus: Implications to Viral Replication and Neuronal Death. PloS One 7, e33871. doi: 10.1371/journal.pone.0033871

Singh, S., Singh, P. K., Suhail, H., Arumugaswami, V., Pellett, P. E., Giri, S., et al. (2020). AMP-Activated Protein Kinase Restricts Zika Virus Replication in Endothelial Cells by Potentiating Innate Antiviral Responses and Inhibiting Glycolysis. J. Immunol. 204 (7), 1810-1824. doi: 10.4049/jimmunol.1901310

Sohail, A., Iqbal, A. A., Sahini, N., Tantawy, M., Winterhoff, M., Ebensen, T., et al. (2021). Itaconate and Derivatives Reduce Interferon Responses and Inflammation. BioRxiv [Preprint]. doi: 10.1101/2021.01.20.427392

Starting, J. R., and van Kuppeveld, F. J. (2017). Viral Rewiring of Cellular Lipid Metabolism to Create Membranous Replication Compartments. Curr. Opin. Cell. Biol. 47, 24-33. doi: 10.1016/j.ceb.2017.02.005

Swain, A., Bambouskova, M., Kim, H., Andhey, P. S., Duncan, D., Auclair, K., et al. (2020). Comparative Evaluation of Itaconate and its Derivatives Reveals Divergent Inflammasome and Type I Interferon Regulation in Macrophages. Nat. Metab. 2, 594-602. doi: 10.1038/s42255-020-0210-0

Tannahill, G. M., Curtis, A. M., Adamik, J., Palsson-McDermott, E. M., McGettrick, A. F., Goel, G., et al. (2013). Succinate Is an Inflammatory Signal that Induces IL- $1 \beta$ through HIF- $1 \alpha$. Nature 496, 238-242. doi: 10.1038/nature11986

Timpani, C. A., and Rybalka, E. (2021). Calming the (Cytokine) Storm: Dimethyl Fumarate as a Therapeutic Candidate for COVID-19. Pharmaceuticals 14, 15. doi: 10.3390/ph14010015

Tonelli, C., Chio, I. I. C., and Tuveson, D. A. (2018). Transcriptional Regulation by Nrf2. Antioxid. Redox Signal. 29, 1727-1745. doi: 10.1089/ars.2017.7342

Vastag, L., Koyuncu, E., Grady, S. L., Shenk, T. E., and Rabinowitz, J. D. (2011). Divergent Effects of Human Cytomegalovirus and Herpes Simplex Virus-1 on Cellular Metabolism. PloS Pathog. 7, e1002124. doi: 10.1371/journal. ppat.1002124

Wang, G. Z., Wang, Y., and Goff, S. P. (2016). Histones Are Rapidly Loaded Onto Unintegrated Retroviral DNAs Soon After Nuclear Entry. Cell Host Microbe 20, 798-809. doi: 10.1016/j.chom.2016.10.009

Williams, N. C., and O'Neill, L. A. J. (2018). A Role for the Krebs Cycle Intermediate Citrate in Metabolic Reprogramming in Innate Immunity and Inflammation. Front. Immunol. 9, 141. doi: 10.3389/fimmu.2018.00141

Wilms, H., Sievers, J., Rickert, U., Rostami-Yazdi, M., Mrowietz, U., and Lucius, R. (2010). Dimethylfumarate Inhibits Microglial and Astrocytic Inflammation by Suppressing the Synthesis of Nitric Oxide, IL-1beta, TNF-Alpha and IL-6 in an 
In-Vitro Model of Brain Inflammation. J. Neuroinflam. 7, 30. doi: 10.1186/ 1742-2094-7-30

Wu, Q., Wang, Q., Mao, G., Dowling, C. A., Lundy, S. K., and Mao-Draayer, Y. (2017). Dimethyl Fumarate Selectively Reduces Memory T Cells and Shifts the Balance Between Th1/Th17 and Th2 in Multiple Sclerosis Patients. J. Immunol. 198, 3069-3080. doi: 10.4049/jimmunol.1601532

Xiao, M., Yang, H., Xu, W., Ma, S., Lin, H., Zhu, H., et al. (2012). Inhibition of $\alpha$ KG-Dependent Histone and DNA Demethylases by Fumarate and Succinate That Are Accumulated in Mutations of FH and SDH Tumor Suppressors. Genes Dev. 26, 1326-1338. doi: 10.1101/gad.191056.112

Yamamoto, M., Yamazaki, S., Uematsu, S., Sato, S., Hemmi, H., Hoshino, K. K., et al. (2004). Regulation of Toll/ IL-1-Receptor-Mediated Gene Expression by the Inducible Nuclear Protein IkappaBzeta. Nature 430, 218-222. doi: 10.1038/ nature 02738

Yu, Y., Clippinger, A. J., and Alwine, J. C. (2011). Viral Effects on Metabolism: Changes in Glucose and Glutamine Utilization During Human Cytomegalovirus Infection. Trends Microbiol. 19, 360-367. doi: 10.1016/ j.tim.2011.04.002

Zhang, J., Zhang, Z., Chukkapalli, V., Nchoutmboube, J. A., Li, J., Randall, G., et al. (2016). Positive-Strand RNA Viruses Stimulate Host Phosphatidylcholine
Synthesis at Viral Replication Sites. Proc. Natl. Acad. Sci. U. S. A. 113, 10641073. doi: $10.1073 /$ pnas. 1519730113

Conflict of Interest: The authors declare that the research was conducted in the absence of any commercial or financial relationships that could be construed as a potential conflict of interest.

Publisher's Note: All claims expressed in this article are solely those of the authors and do not necessarily represent those of their affiliated organizations, or those of the publisher, the editors and the reviewers. Any product that may be evaluated in this article, or claim that may be made by its manufacturer, is not guaranteed or endorsed by the publisher.

Copyright (c) 2021 Sánchez-García, Pérez-Hernández, Rodríguez-Murillo and Moreno-Altamirano. This is an open-access article distributed under the terms of the Creative Commons Attribution License (CC BY). The use, distribution or reproduction in other forums is permitted, provided the original author(s) and the copyright owner(s) are credited and that the original publication in this journal is cited, in accordance with accepted academic practice. No use, distribution or reproduction is permitted which does not comply with these terms. 